\title{
Alarm calls as costly signals of antipredator vigilance: the watchful babbler game
}

\author{
CARL T. BERGSTROM* \& MICHAEL LACHMANN† \\ *Department of Biology, Emory University \\ $\dagger$ Santa Fe Institute, Santa Fe
}

(Received 28 January 2000; initial acceptance 9 March 2000;

final acceptance 10 August 2000; MS. number: A8703)

\begin{abstract}
Alarm-calling behaviour is common in many species that suffer from predation. While kin selection or reciprocal altruism are typically invoked to explain such behaviours, several authors have conjectured that some alarm calls may instead be costly signals sent by prey to inform approaching predators that they have been detected. We develop a general game-theoretical model, the watchful babbler game, in which prey signal awareness to predators. We derive necessary and sufficient conditions for alarm calls to function as honest signals. We show that signals can honestly reveal prey awareness if (1) the prey's sense of predation risk accurately reflects the probability that the predator is present, and (2) greater awareness of the predator allows the prey a greater chance of escape. When honest signalling is possible, the model predicts that prey will be more willing to signal when predators are common than when predators are rare, and that greater pursuit costs to the predator will allow cheaper signals by the prey.
\end{abstract}

() 2001 The Association for the Study of Animal Behaviour

Alarm-calling behaviour has provided a persistent puzzle to behavioural biologists. Why should an individual risk its own survival to produce costly 'warnings' of a predator's presence (Maynard Smith 1965)? Evolutionary biologists have typically invoked kin selection and/or reciprocal altruism to explain such behaviours (reviewed by Hauser 1996, pp. 413-433). While a calling individual may suffer some direct cost, it may also reap inclusive fitness benefits by warning relatives of danger, or anticipate direct fitness benefits from future reciprocity.

However, several authors have argued that alarm calls may serve an alternative function (Perrins 1968; Smythe 1970; Zahavi 1977, 1987; Woodland et al. 1980). Calls may not be directed to conspecifics, but instead to the predators themselves. Hasson (1991) and Caro (1995) review numerous empirical studies testing this class of hypotheses, and examine the role of signal cost in ensuring signal honesty under such circumstances.

The basic logic of these explanations is as follows. Consider a prey individual that is for some reason an 'undesirable' target for a predator. Thus, both the predator and the prey have a common interest in communicating information about the prey's 'undesirability'. By

Correspondence and present address: C. T. Bergstrom, Department of Zoology, University of Washington, Box 351800, Seattle, WA 981951800, U.S.A. (email: cbergst@u.washington.edu). M. Lachmann is at the Santa Fe Institute, 1399 Hyde Park Road, Santa Fe, NM 875018943, U.S.A.

sharing this information, the prey avoids pursuit; by receiving it the predator avoids wasting time and energy in the chase. A prey individual may be 'undesirable' for any of a number of reasons: it may be particularly fast and skilled at escape, it may be particularly dangerous once caught, it may be of relatively low nutritional value, or it may already be aware of the predator's presence and thus more likely to escape (or to cause harm to the attacking predator) than an unsuspecting prey individual (Hasson 1991). Previous theoretical work has focused on the first of these reasons, examing 'quality advertisement' models in which prey of varying qualities use costly signals to convey their skill at evading pursuit (Nur \& Hasson 1984; Vega-Redondo \& Hasson 1993; Yachi 1995). Motivated by the growing literature on signalling of awareness, we focus in this paper on the last of these possibilities: a prey individual is confronted with imperfect information about the predator's presence, and signals its 'awareness of the predator' rather than its strength, speed, or other physiological correlates of escape ability.

Putative signals of awareness from prey to predator have been described in kangaroo rats (Randall \& Stevens 1987; Randall \& Matocq 1997), deer (Caro et al. 1995), lizards (Leal \& Rodriguez-Robles 1997; Leal 1999), guppies (Godin \& Davies 1995), skylarks (Cresswell 1994), tetras (Brown et al. 1999), primates (Zuberbühler et al. 1999), and numerous other species from a diverse range of taxa (Caro 1995). For illustration, we detail here an 
avian example presented by Zahavi \& Zahavi (1997, Chapter 1), describing several curious features of alarmcalling behaviour in babblers. When a member of a feeding flock spies a potentially dangerous predator, it lets out a shrill 'barking' alarm call; the others in the flock may initially dive for cover but often the entire flock joins the initial signaller atop the tree, all loudly issuing alarm calls. As Zahavi \& Zahavi point out, this behaviour is not easily explained by the conspecific-warning theories of alarm calling. If the intended receivers of the alarm calls are the other flock members, why do the birds continue calling long after the entire flock has assembled and joined the barking? Why do the babblers employ such a loud alarm call, which may attract the attention of the predator in question, or even the attention of yet unseen predators, when a much softer call would seemingly be sufficient to alert the members of the flock?

Zahavi \& Zahavi conjecture that the answer to these questions is that the alarm calls are actually directed to the predator, rather than to conspecifics. By barking loudly, the babblers inform the predator, say, a raptor, that it has been seen, and that any attack will probably be unsuccessful. Sharing this information is to the advantage of both predator and prey. The raptor does well moving on, and waiting for a chance at unsuspecting prey; the babblers are better off avoiding the attack even if they are aware of the raptor beforehand (Zahavi \& Zahavi 1997).

If alarm calls actually deter the raptor's attack, why would a babbler not gain from calling indiscriminately, even before sighting a predator, on the off-chance that one is present? Zahavi \& Zahavi offer an answer to this puzzle as well: 'A babbler who would cheat by going to the top of the canopy and barking before it saw a predator would expose itself to raptors it might not have noticed. That risk helps ensure that if a babbler goes to the top of the tree and declares it has seen a raptor, it has indeed seen one' (Zahavi \& Zahavi 1997, page 5). That is to say, if the signal of awareness is sufficiently costly, prey will benefit from sending this signal only when they are quite certain that they have spotted a predator.

While this verbal argument seems plausible, it is difficult to evaluate without a formal mathematical model. Similarly, to qualify as a proper hypothesis, the predatordeterrence explanation for alarm calling must make clear predictions (ideally, as specific as possible) based on some underlying model of prey-to-predator signalling (Caro 1995). Under what circumstances, if any, will this costly signalling mechanism allow honest communication of awareness from prey to predator? And how are changes in the environment (e.g. changes in the prevalence of predators, the cost of pursuit, availability of alternative prey, or the cost of signalling) expected to affect the frequency of alarm calls?

Here, we develop a game theoretical model of the interaction between a single babbler and an aerial predator, which we call the watchful babbler game. (We leave for future consideration the full range of social motives and interactions that become possible when not one but rather a whole flock of prey individuals is present.) We explore the conditions under which alarm calls can function as honest signals from prey to predator in this game. In the following section, we describe the structure of this game. We then find the necessary conditions for existence of an honest signalling equilibrium (see Analysis), and consider how changes in the system will affect the equilibrium signalling behaviour. Finally, we describe the predictions made by the model, discuss the general conclusions and consider possible extensions (see Discussion).

\section{THE WATCHFUL BABBLER GAME}

A small bird is feeding alone in the bushes when it catches a glimpse of something soaring overhead. A hawk? A heron? A falcon? A shadow? A simple trick of the light? Sometimes the small bird, which we will call the 'prey', gets a good look before its view is obstructed by the bushes and trees of the forest. Other times, it merely catches a glimpse of a swiftly moving form in the sky.

In either case the prey can attempt to deter pursuit by issuing an alarm call, loudly proclaiming that it has seen the predator (and, thus, implying that it has the knowledge and forewarning needed to escape; see below). However, such a signal is not without cost. The act of signalling takes time and energy, and exposes the prey to detection and potential pursuit by other 'secondary' predators. There are no conspecifics nearby for the prey to warn (or to warn the prey, for that matter), so the only function of a signal would be to deter predation. If the 'primary' predator is actually present in the vicinity of the prey, this predator takes into account any alarm call that may have been sent, and decides whether or not to pursue the prey.

Of course, no two prey-predator interactions will be precisely the same. The sensory stimuli observed by the prey will vary from moment to moment. Sometimes the prey will perceive a high likelihood of attack by a predator; other times the prey will not realize that a predator is nearby. Furthermore, some situations will afford the predator a better chance of catching the prey than others; the predator's chance of successful pursuit will naturally vary from one interaction to the next.

To take these variations into account, we draw two random variables at the beginning of each iteration of the game. The stimuli observed by the prey are summarized by a random variable $x$. Since these stimuli may depend on whether the predator is present, $x$ is drawn from one distribution when the predator is absent and from a separate (but overlapping) distribution when the predator is present. The probability that the predator will capture the prey should it choose to give chase is specified by a random variable $t$. Because we might expect the observed stimuli to be in some way correlated with the probability of successul pursuit, $x$ and $t$ will be drawn together from a bivariate distribution when the predator is present.

The full game, shown in extended form in Fig. 1, proceeds as follows.

(1) In a given iteration of the game, the predator is present in the vicinity of the prey (hereafter, 'present') with probability $\alpha$, and absent from the vicinity of the 
(1) Nature chooses whether predator is present

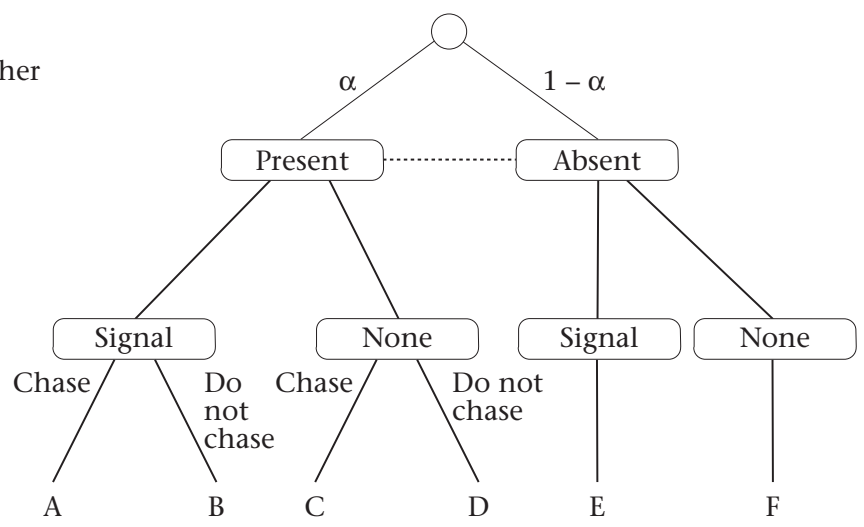

Figure 1. Extended form of the watchful babbler game. (1) 'Nature' decides at random whether or not the predator is present in the vicinity of the prey, and then selects random values for $x$ and, if the predator is present, for $t$. (For simplicity, the choice of $x$ and $t$ is not shown in the diagram.) (2) The prey assesses the situation. It cannot tell for certain whether the predator is present or absent, but it does observe stimuli associated with predation risk, summarized by $x$. Given $x$, it chooses an action: signal, or do not signal. (3) If present, the predator observes whether or not a signal has been sent, but cannot directly assess its chance of catching the prey, $t$, or even the prey's observations, $x$. Based on the prey's signal alone, the predator chooses an action of its own: chase, or do not chase. The final payoffs for each outcome (A-F) are summarized in Table 1.

Table 1. Payoffs to prey and predator

\begin{tabular}{|c|c|c|c|c|c|c|}
\hline & \multicolumn{4}{|c|}{ Predator present } & & \\
\hline & \multicolumn{2}{|c|}{ Signal } & \multicolumn{2}{|c|}{ No Signal } & \multicolumn{2}{|c|}{ Predator absent } \\
\hline & Chase & Do not chase & Chase & Do not chase & Signal & No signal \\
\hline Outcome & A & B & $C$ & D & $\mathrm{E}$ & $\mathrm{F}$ \\
\hline Prey & $(1-c)(1-t)$ & $1-c$ & $1-t$ & 1 & $1-c$ & 1 \\
\hline Predator & $t-d$ & 0 & $t-d$ & 0 & 0 & 0 \\
\hline
\end{tabular}

See Fig. 1, Appendix 1.

prey (hereafter, 'absent') with probability $1-\alpha$. If the predator is present, the stimuli observed by the prey, $x \in[0,1]$, and the probability that a chase would be successful, $t \in[0,1]$, are drawn from a joint distribution $F$. If the predator is absent, it of course has no chance of catching the prey. The prey may still observe various stimuli and imagine it has seen a predator; to reflect this circumstance, $x$ is then drawn from another distribution $G$.

(2) Based on $x$, the prey decides whether or not to send a signal to inform the predator that it has been seen. This signal is costly; with some probability it will attract the attention of a 'secondary' predator that might itself pursue the prey. We assume that this secondary predation risk can be summarized by a multiplicative signal cost $c$. Although the prey's choice of whether or not to send the signal may affect the primary predator's choice of whether or not to pursue, this choice does not affect the chance $t$ that the primary predator will be successful if it does pursue.

(3) If the predator is absent, the game is over. If the predator is present, it obseves the prey's signal (but note that it cannot directly observe $x$ or $t$ ) and decides whether or not to engage in a chase, with a gain of 1 if successful and a cost of $d$ regardless of whether the chase is successful. The final payoffs of the game are given in Table 1.

\section{Analysis}

We begin by determining whether and when a signalling equilibrium exists in the watchful babbler game. That is, when is there an equilibrium at which some prey signal, others do not, and predators chase only the nonsignalling prey? (There will not be an equilibrium where predators only chase the signalling prey, because if predators behaved in this way, then no prey would benefit from signalling at equilibrium.) At a signalling equilibrium, prey are willing to signal if and only if the expected benefit of deterring pursuit exceeds the cost $c$ of signalling. To express this mathematically, we require a few definitions. Let the distribution $F$ have joint probability density $f(t, x)$ and marginal densities $f_{T}(t)=\int_{O}^{1}(t, x) d x$ and $f_{X}(x)=\int_{0}^{1}(t, x) d t$, respectively. Let $G$ have probability density $g(x)$. Notice that an individual prey can use its observed predation risk $x$ to 'estimate' the chance $t$ that it will be captured if pursued; define $t^{\star}(x)$ to be this expected value of $t$ given $x$ when the predator is present. Similarly the prey can estimate the probability that the predator truly is present; define $p(x)$ to be the probability that the 
predator is present conditional on $x$. A bit of algebra reveals that, when the prey is pursued, the probability that it will actually be caught is simply the product of $p(x)$ and $t^{*}(x)$; define $\psi(x)$ to be this product. We can order the observed predation risks $x$ freely; therefore without loss of generality we can assume that $\psi(x)$ is a monotone increasing function. In terms of the parameters and probability density functions defined above, these definitions can be written as follows:

$$
\begin{aligned}
\operatorname{Pr}[\text { present } \mid x] & \equiv p(x) \\
& =\frac{\alpha f_{X}(x)}{\alpha f_{X}(x)+(1-\alpha) g(x)}
\end{aligned}
$$

$\operatorname{Pr}[$ capture $\mid$ present and $x] \equiv t^{*}(x)$

$$
\begin{aligned}
& =\frac{\int_{0}^{1} f(t, x) t d t}{f_{X}(x)} \\
\operatorname{Pr}[\text { capture } \mid x] & \equiv \psi(x) \\
& =p(x) t^{*}(x) .
\end{aligned}
$$

At a signalling equilibrium, a signal always deters pursuit and therefore the expected payoff to the prey conditional on $x$ is $1-c$ if it signals, and $1-\psi(x)$ if it does not. At equilibrium the prey will signal if and only if $c<\psi(x)$. We can use this fact to partition the prey into two sets: a signalling pool and a nonsignalling pool. Label a prey individual that observes $x_{i}$ as 'prey $x_{i}{ }^{\prime}$. Divide the set of all prey into two subsets: $X_{+}$, those that at a signalling equilibrium have a higher expected payoff from signalling or are indifferent between signalling and not signalling, and $X_{-}$, those that at a signalling equilibrium have a higher expected payoff from not signalling. Then,

$c \leq \psi(x)$ for all values of $x$ in $X_{+}$ $c>\psi(x)$ for all values of $x$ in $X_{-}$.

The signal cost $c$ and the shape of the function $\psi(x)$ now determine which prey should signal and which should remain silent (i.e. they determine the bounds of $X_{+}$and $X_{-}$, respectively). To have an informative signalling equilibrium, in which some but not all prey signal, we require $\psi(0)<c<\psi(1)$. From this, two results follow. First, since $\psi(0)$ and $\psi(1)$ are probabilities, the cost $c$ must be strictly positive for this inequality to hold. Therefore the signals of awareness must be costly, in the present model, if there is to be an informative signalling equilibrium. Second, since the function $\psi$ is monotone, it will cross $c$ at most once. Therefore a signalling equilibrium will always have a threshold level of observed predation risk $x$ above which prey signal and below which they do not. This threshold, which we call $\hat{x}$, is given by $\hat{x}=\psi^{-1}(c)$. An example is shown in Fig. 2 .

We now consider the predator's behaviour. At a signalling equilibrium, the predator will heed the prey's signal, chasing if and only if no signal is sent. Therefore, the predator must have a higher expected payoff from pursuing the prey if and only if no signal is sent. We denote

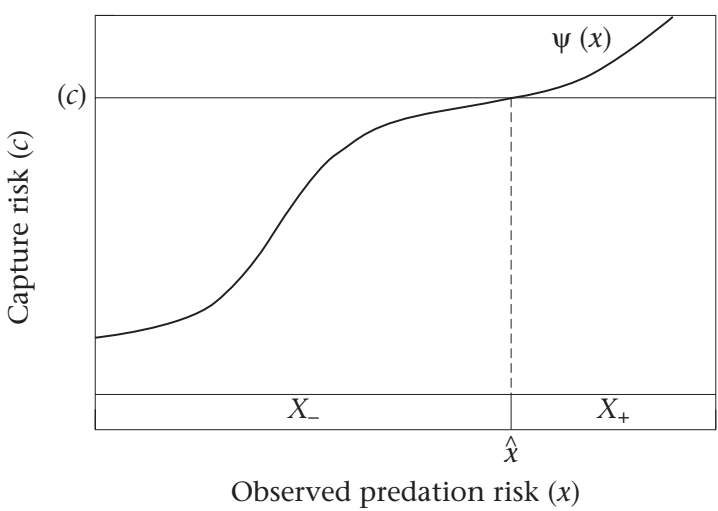

Figure 2. The signal cost $c$ and the shape of the observed predation risk curve $\psi(x)$ determine the range of signalling prey $X_{+}$and the range of nonsignalling prey $X_{-}$.

a pursuing predator's average probability of capturing prey that observe predation risks above the signalling threshold as $t_{+}^{\diamond}(\hat{x})$, and the average probability of capturing prey that observe risks below the threshold as $t_{-}^{\diamond}(\hat{x})$, where $\hat{x}$ is the signalling threshold as defined above.

$$
\begin{aligned}
& t_{+}^{\diamond}(\hat{x}) \equiv \frac{\int_{\hat{x}}^{1}\left(\int_{0}^{1} f(t, x) t d t\right) d x}{\int_{\hat{x}}^{1} f_{X}(x) d x} \\
& t_{-}^{\diamond}(\hat{x}) \equiv \frac{\int_{0}^{\hat{x}}\left(\int_{0}^{1} f(t, x) t d t\right) d x}{\int_{0}^{\hat{x}} f_{X}(x) d x}
\end{aligned}
$$

The values of $t_{+}^{\diamond}$ and $t_{-}^{\diamond}$ can be seen as analogues of the value $t^{*}$. All are estimates of capture probability, but $t^{*}$ represents the prey's point of view whereas the $t^{\diamond}$ values represent the predator's point of view. The prey knows the precise value of $x$ and so capture probability $t^{*}$ is simply an integral over the possible values of $t$ for that particular value of $x$. By contrast, the predator only knows that $x$ is in some range and therefore the $t^{\diamond}$ values require a double integral over all values of $t$ for each value of $x$.

The expected payoff to a pursuing predator is the conditional expectation $t^{\diamond}$ minus the cost of pursuit $d$. The payoff to a nonpursuing predator is always 0 . The signalling equilibrium conditions on the predator's payoffs are therefore as follows:

$$
t_{+}^{\diamond}(\hat{x})>d>t_{-}^{\diamond}(\hat{x}) .
$$

Signalling equilibrium requires that

$$
\frac{\int_{0}^{\hat{x}} f_{X}(x) t^{*}(x) d x}{\int_{0}^{\hat{x}} f_{X}(x) d x}>\frac{\int_{\hat{x}}^{1} f_{X}(x) t^{*}(x) d x}{\int_{\hat{x}}^{1} f_{X}(x) d x}
$$

Our first result follows directly. 


\section{Result 1}

A necessary condition for the existence of a signalling equilibrium is the following: prey that observe predation risks above the signalling threshold are, on average, less likely to be caught if pursued than are prey that observe predation risks below the signalling threshold.

Clearly not all prey-predator systems will have this property. In some cases (sessile prey organisms providing an extreme example) the prey's knowledge and forewarning of the predation risk will have little or no impact on the probability of capture should the predator elect to pursue. The implication here is that the prey are somehow able to use their knowledge and forewarning of the predation risk (e.g. of the predator's presence and/or location) to increase their chance of escape should they be pursued.

Result 1 states the necessary connection between the observed predation risk $\psi(x)$ and the probability of capture if pursued $t^{\star}(x)$. In Appendix 2 we derive a similar result for the connection between $\psi(x)$ and the probability that the predator is present $p(x)$ :

$$
\frac{\int_{0}^{\hat{x}} f_{X}(x) / p(x) d x}{\int_{0}^{\hat{x}} f_{X}(x) d x}>\frac{\int_{\hat{x}}^{1} f_{X}(x) / p(x) d x}{\int_{\hat{x}}^{1} f_{X}(x) d x}
$$

\section{Result 2}

A second necessary condition for signalling equilibrium is the following: prey that observe predation risks above the signalling threshold have a lower average value of $1 / p(x)$ than do those observing risks below the threshold, where $p(x)$ is the probability that the predator is present given the observed predation risk $x$.

Roughly speaking, because $\psi$ is increasing in $x$ and $t^{*}$ is decreasing on average (Result 1), their quotient $p$ must be increasing on average. This means that prey that are less frightened of predation must be less certain of the predator's presence if a signalling equilibrium is to exist. (More precisely, the actual condition states that the average inverse of $p$ is greater in the nonsignalling pool than in the signalling pool, which usually but not always will mean that the average of $p$ itself will be greater in the signalling pool.)

Results 1 and 2 provide necessary conditions for a signalling equilibrium. To establish that such an equilibrium can exist at all, we derive a set of sufficient conditions in Appendix 3.

Condition (6) above specifies the requirements necessary to provide the predator with the incentive to heed the prey's signal. However, only one of the two inequalities in (6) will be binding for any given set of parameters. We can see this by considering two cases separately: (A) cases in which the predator would pursue in the absence of any information and (B) cases in which the predator would not pursue in the absence of any information. We note that the average $t^{*}$ value over all prey will be $t_{+}^{\diamond}(0)=t_{-}^{\diamond}(1)$. When this value exceeds $d$, we have case A; when this value is less than $d$, we have case B. For the remainder of this paper, we will assume that $t(x)$ and $p(x)$ are continuous and monotone (decreasing and increasing respectively) over $(0,1)$; then the functions $t_{-}^{\diamond}$ and $t_{+}^{\diamond}$ can be inverted. While these assumptions simplify the analysis considerably, they are not essential to the general line of argument.

We begin with case A. Since the functions $t_{+}^{\diamond}$ and $t_{-}^{\diamond}$ and the value of $\hat{x}$ are determined by $F, G$ and $c$, the expression (6) can be seen as a necessary condition on $d$ for the existence of a signalling equilibrium. Alternatively, (6) can be seen as a condition of feasible $\hat{x}$ values (and ultimately $c$ values) given $d, F$ and $G$. We take the latter approach in Fig. 3 and in the exposition that follows. The top half of Fig. 3a illustrates the necessary condition on $\hat{x}$ in case A. In order that (6) is met, we require that $d>t_{+}^{\diamond}(\hat{x})$, or

$$
\hat{x}>\underline{x} \text {, where } \underline{x} \equiv t_{+}^{\diamond-1}(d)
$$

Now, we can use this range of feasible $\hat{x}$ values to determine how much signalling must cost. The value of $\hat{x}$ is determined by $\psi$ and $c$ alone: $\hat{x}=\psi^{-1}(c)$. Expression (9) then becomes $\psi(\underline{x})<c$. Of course, some prey must signal so we also require $c<\psi(1)$. As shown in the bottom of Fig. 3a, our final condition on cost $c$ becomes

$$
\psi(\underline{x})<c<\psi(1)
$$

A similar analysis can be applied to case B. In this case, in order to meet (6) it must be that $d<t_{-}^{\diamond}(\hat{x})$, or equivalently

$$
\hat{x}<\bar{x} \text {, where } \bar{x} \equiv t_{-}^{\diamond}-1(d)
$$

This is shown in the top half of Fig. 3b. As before, we can now find necessary conditions on $c$ such that some but not all prey signal and (11) holds:

$$
\psi(\bar{x})>c>\psi(0)
$$

This is illustrated in the bottom half of Fig. 3b. Of course, we could reverse any of this analysis, to find necessary conditions on the cost of pursuit $d$, given $c$.

In case $B$ the signalling equilibrium exists only because the prey themselves make pursuit worthwhile by the act of signalling, an act that helps the predator to identify the most vulnerable prey. Ironically, in this case no prey are better off (and no predators are worse off) in the signalling equilibrium than in the nonsignalling equilibrium. We can imagine at least two ways for evolution to lead to such a situation. First, if prey signal to one predator (e.g. hawks) that would pursue in the absence of information and if prey cannot easily distinguish these from a second predator (e.g. eagles) that would not pursue in the absence of information, the act of signalling to deter predation by the former (hawks) will facilitate predation by the latter (eagles). Second, suppose predators initially would chase in the absence of information. Signalling may evolve to the mutual benefit of prey and predator. If the parameters of the system then somehow change, so pursuit in the absence of information is no longer worthwhile (e.g. if an alternative food source becomes more 
(a)
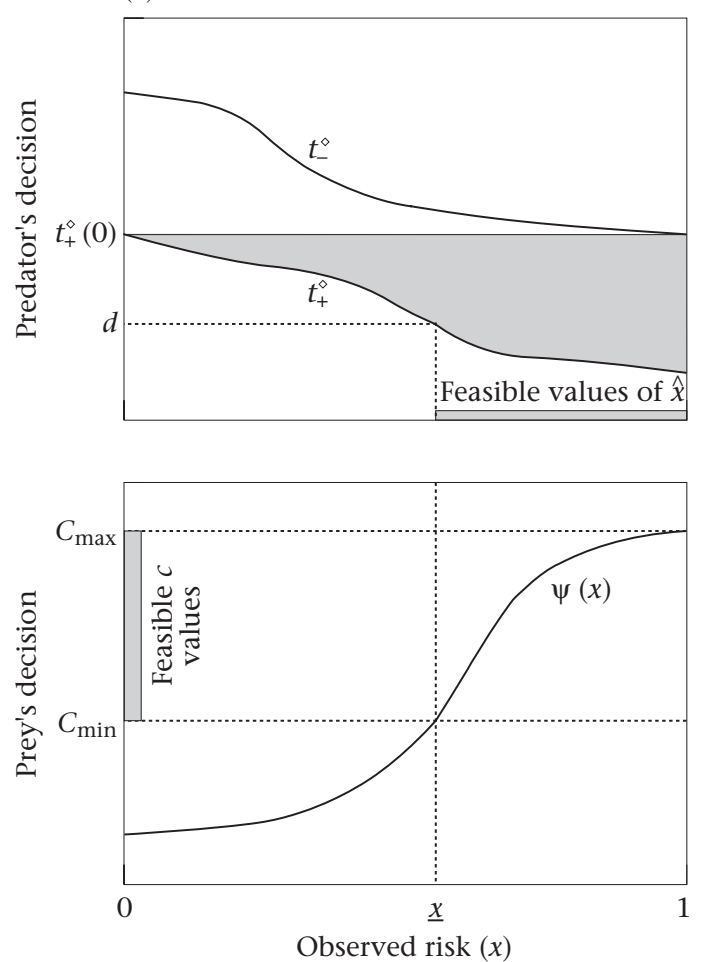

(b)
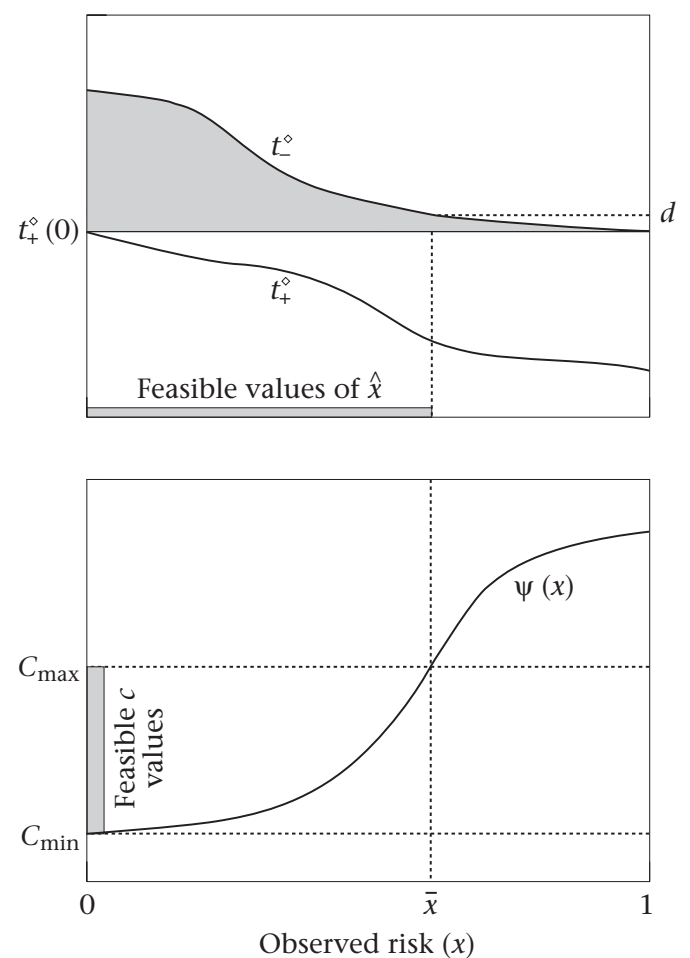

Figure 3. (a) The predator would pursue in the absence of any information. Here, the predator will heed the prey's signal only if the signalling threshold $\hat{x}$ exceeds a minimum signalling threshold $\underline{x}$. This minimum signalling threshold is the level of observed risk $x$ at which the pursuit cost $d$ equals the probability of capturing a signalling prey individual, $t_{+}^{\diamond}(\hat{x})$. As depicted in Fig. 2 , the signal cost $c$ and the distributions $F$ and $G$, via the function $\psi$, determine the actual signalling threshold $\hat{x}$ used by the prey. In order to have a signalling equilibrium, we require $\underline{x}<\hat{x}<1$. (b) The predator would not pursue in the absence of any information. Here, the predator will heed the prey's signal only if the signalling threshold $\hat{x}$ is less than some maximum $\bar{x}$. This maximum signalling threshold is the level of observed risk $x$ at which the pursuit cost $d$ equals the probability of capturing a nonsignalling individual. Again, signal cost and the distributions $F$ and $G$ determine the actual signalling threshold $\hat{x}$ used by the prey. Signalling equilibrium requires $0<\hat{x}<\bar{x}$.

abundant, effectively increasing the cost of pursuit $d$ ), the prey will not necessarily be able to leave the signalling equilibrium for the nonsignalling equilibrium and therefore may get trapped at the signalling equilibrium (see Bergstrom \& Lachmann 1997 for a similar example in signalling among relatives).

In addition, we can ask how the parameters of the model affect the equilibrium signalling threshold $\hat{x}$, the minimum signalling threshold $\underline{x}$ for which the predator would heed the signal in case $A$, and the maximum signalling threshold $\bar{x}$ for which the predator would heed the signal in case $B$. These effects are summarized in Table 2 .

The first column of Table 2 indicates that as $\alpha$ increases (e.g. as predators become more common in a local

Table 2. The change in $\hat{x}, \underline{x}$ and $\bar{x}$ as $\alpha, d$ and $c$ are increased

\begin{tabular}{|c|c|c|c|}
\hline Parameter & $\hat{x}$ & $\underline{x}$ & $\bar{x}$ \\
\hline$\alpha$ & Decreases & No change & No change \\
\hline$d$ & No change & Decreases & Decreases \\
\hline c & Increases & No change & No change \\
\hline
\end{tabular}

See text and Appendix 1. environment), $\hat{x}$ decreases, and thus more prey would be willing to signal at a given cost $c$. Alternatively, since the relation $\psi$ between $\hat{x}$ is monotone increasing, we can say that the signal cost $c$ necessary to maintain a given threshold $\hat{x}$ increases as $\alpha$ increases. When $\alpha$ is held constant, by contrast, the signalling threshold $\hat{x}$ will increase as signal cost $c$ increases.

The second column of Table 2 shows what happens to $\underline{x}$ (in case $\mathrm{A}$; in case $\mathrm{B}, \underline{x}$ is neither well defined nor relevant) as parameters of the system change. As the pursuit cost $d$ increases, the minimum feasible signalling threshold $\underline{x}$ decreases. Again because of the monotonicity of $\psi$, this implies that signalling can be maintained with less expensive signals when pursuit costs increase (either because the actual pursuit cost increases or because the opportunity cost increases due to the changing availability of other food sources.) Therefore we would predict that when pursuit is costly, signalling can be cheap and prey can afford to signal at every little rustle in the bushes. When pursuit is inexpensive, by contrast, signalling will necessarily be very costly and will only be employed when the prey is relatively certain that the predator is actually present. Similarly, the third column indicates that as pursuit cost $d$ increases, the maximum signalling threshold in case $\mathrm{B}, \bar{x}$, decreases. 


\section{DISCUSSION}

Our analysis shows that, as suggested by Zahavi and others, the costly signalling mechanism can indeed stabilize honest signalling of awareness between prey and predator. However, stable signalling requires that prey that are less likely to be caught are none the less the ones willing to signal, because they consider themselves to be at greater risk. This sounds contradictory; how could this happen unless prey were misinterpreting their sensory stimuli? (At equilibrium prey will not be misinterpreting stimuli; if they were, they could be invaded by prey that correctly used that information.)

The answer lies in the fact that the prey do not know for certain whether or not the predator is present. Therefore prey that are hard to catch from the predator's perspective may at the same time consider themselves to be at grave risk, if the prey's degree of concern takes into account its own estimate of the probability that the predator is actually present. If the prey that are more certain of a predator's presence (and thus willing to invest in deterring pursuit) are also those that the predators do not wish to pursue, a signalling equilibrium is possible.

In the section above, we derived two necessary conditions for the existence of a signalling equilibrium.

(1) The prey's 'sense' of the predation risk must accurately reflect the chance that a predator is present. That is, prey that are more concerned about predation must be more likely to have a predator in the vicinity.

(2) Prey that sense that they are at risk of predation must be less likely to be caught if actually pursued by a predator than those that are unaware of the predation threat.

Taken together, the two requirements above imply that prey that are more aware of the predator's presence must have a better chance of escape. That is, there must be a strong inverse relationship between the prey's certainty that a predator is present and the prey's chance of being captured if pursued. The prey's information about the predator's presence must be helpful in evading predation should pursuit occur. This should hold in many predatorprey systems; should pursuit occur, more aware prey will often get a quicker jump in fleeing from the predator, and will be better able to choose an effective escape route. The model also makes a set of predictions, summarized in Table 2, for how signal cost and signal prevalence will change as a function of the environmental parameters. Of particular interest are the observations that (1) more prey will be willing to signal when predators are common than when predators are rare and (2) greater pursuit costs to the predator will allow cheaper signals by the prey, and vice versa.

As mentioned in the introduction, our model considers the case in which prey signal their awareness of the predator rather than their physiological ability to escape pursuit. There are other differences as well. In previous models (Nur \& Hasson 1984; Vega-Redondo \& Hasson 1993) the cost of signalling is imposed by the predator for whom the signal is intended. In our model, the cost of signalling is imposed by other predators that may be alerted to the prey's location by the signals. Therefore, in previous models, a signal increases the chance of capture if pursued by the 'primary' predator, but the signal cost is paid only if the primary predator chooses to pursue. In the watchful babbler game, the signal itself does not alter the chance of capture by the primary predator should it choose to pursue, but the signal cost is paid whether or not the primary predator chooses pursuit.

We have seen that at the signalling equilibrium, the prey with the greatest observed risk of predation $\psi(x)$ always signal. However, it does not follow that the prey that are most certain of the predator's presence will always signal. Indeed, if the risk of capture $t^{\star}(x)$ drops sufficiently for prey that are certain of the predator's presence (i.e. for prey that have a high $p(x)$ value), then these prey will not signal even though they 'know' that the predator is present. This provides an additional testable prediction. The model predicts that in some systems, prey that get a particularly early and/or informative look at an approaching predator will simply sneak away to safety instead of signalling. Such behaviour is not expected under kin-selection theories of alarm calling, and thus if observed, would give us reason to suspect that alarm calls are signals intended for the predator (and perhaps conspecifics as well) rather than for conspecifics alone.

Although we allow the prey to estimate the capture probability $t$ via $x$, we have simplified the present model by assuming that the predator has no interaction-specific information with which to make its own estimate of $x$, other than the prey's signal. What would happen if the predator also had an estimate, call it $y$, of $t$ ?

In this case, different individual predators would have different 'presignal' estimates of $t$, and therefore would require different degrees of convincing before deciding not to chase. Prey will then have cause to employ more than one signal. Prey that are quite certain of the predator's presence will be willing to invest in expensive signals that will deter as many predators as possible. Prey that are less certain will only be willing to invest in relatively inexpensive signals that merely deter those predators that were already near to indifferent. Readers familiar with the Sir Philip Sidney game (Maynard Smith 1991) will see the parallel between this extension of the watchful babbler game and the Sir Philip Sidney extension detailed by Johnston \& Grafen (1992). As in the Sir Philip Sidney game, many signalling equilibria should be possible in this watchful babbler extension, including a fully separating equilibrium in which each different prey individual sends a distinct signal, and a plethora of pooling equilibria in which some prey share common signals (Lachmann \& Bergstrom 1998). However, in contrast to the Sir Philip Sidney game, the watchful babbler game will not allow uniformly costfree signals (Bergstrom \& Lachmann 1998), because all prey would like to be seen as more alert than they truly are. In the absence of signal cost, prey would be selected to signal awareness in every 'play' of the game, whether or not they have truly spotted the predator. Under those circumstances, the signals would not remain honest and there would soon be no reason for the predators to heed them. 
We would like to conclude with a caveat regarding the generality of our model. We have chosen to treat the predator-prey interaction as a two-player game, whereas many if not most such interactions will in practice occur in the presence of other individuals. This of course complicates matters; signals that are primarily directed to the predator may also serve as sexual selection signals (Cressewll 1994; Godin \& Dugatkin 1996; Leal 1999), warnings to kin (Blumstein \& Armitage 1997), or signals of status (Zahavi 1990). None the less, we feel that our model captures the crucial axis of interaction, and as such should be useful in clarifying the mechanism and requirements of the predator-prey hypothesis for alarm-call signalling.

\section{Acknowledgments}

The main ideas for this paper arose during a series of discussions with R. Johnstone, G. Nöldeke and L. Samuelson. The authors wish to thank T. Bergstrom, G. Nöldeke and L. Samuelson for many helpful discussions and for their extensive commentary on the manuscript. D. Blumstein and an anonymous referee also provided valueable perspective and numerous suggestions for improving the exposition. C. Bergstrom was partially supported by NIH/NIAID grant T32-AI0742. M. Lachmann is supported by the Santa Fe Institute.

\section{References}

Bergstrom, C. T. \& Lachmann, M. 1997. Signalling among relatives. I. Is costly signalling too costly? Philosophical Transactions of the Royal Society of London, Series B, 352, 609-617.

Bergstrom, C. T. \& Lachmann, M. 1998. Signalling among relatives. III. Talk is cheap. Proceedings of the National Academy of Sciences, U.S.A., 95, 5100-5105.

Blumstein, D. T. \& Armitage, K. B. 1997. Does sociality drive the evolution of communicative complexity? A comparative test with ground-dwelling sciurid alarm calls. American Naturalist, 150, 179-200.

Brown, G. E., Godin, J. C. J. \& Pedersen, J. 1999. Fin-flicking behaviour: a visual antipredator alarm signal in a characin fish, Hemigrammus erythrozonus. Animal Behaviour, 58, 469-475.

Caro, T. M. 1995. Pursuit-deterrence revisited. Trends in Ecology and Evolution, 10, 500-503.

Caro, T. M., Lombardo, L., Goldizen, A. W. \& Kelly, M. 1995 Tail-flagging and other antipredator signals in white-tailed deer: new data and synthesis. Behavioral Ecology, 6, 442-450.

Cresswell, W. 1994. Song as a pursuit-deterrent signal, and its occurrence relative to other anti-predation behaviours of skylark (Alauda arvensis) on attack by merlins (Falco columbarius). Behavioral Ecology and Sociobiology, 34, 217-223.

Godin, J. G. J. \& Davis, S. A. 1995. Who dares, benefits: predator approach behavior in the guppy (Poecilia reticulata) deters predator pursuit. Proceedings of the Royal Society of London, Series $B$, 259, 193-200.

Godin, J. G. J. \& Dugatkin, L. A. 1996. Female mating preference for bold males in the guppy Poecilia reticulata. Proceedings of the National Academy of Sciences, U.S.A., 93, 10262-10267.

Hasson, O. 1991. Pursuit deterrent signals: the communication between prey and predator. Trends in Ecology and Evolution, 6, 325-329.
Hauser, M. D. 1996. The Evolution of Communication. Cambridge, Massachusetts: MIT Press.

Johnstone, R. A. \& Grafen, A. 1992. The continuous Sir Philip Sidney game: a simple model of biological signalling. Journal of Theoretical Biology, 156, 215-234.

Lachmann, M. \& Bergstrom, C. T. 1998. Signalling among relatives. II. Beyond the Tower of Babel. Theoretical Population Biology, 54, 146-160.

Leal, M. 1999. Honest signalling during prey-predator interactions in the lizard Anolis cristatellus. Animal Behaviour, 58, 521-526.

Leal, M. \& Rodriguez-Robles, J. A. 1997. Signalling displays during predator-prey interactions in a Puerto Rican anole, Anolis cristatellus. Animal Behaviour, 54, 1147-1154.

Maynard Smith, J. 1965. The evolution of alarm calls. American Naturalist, 99, 59-63.

Maynard Smith, J. 1991. Honest signalling: the Philip Sidney game. Animal Behaviour, 42, 1034-1035.

Nur, N. \& Hasson, O. 1984. Phenotypic plasticity and the handicap principle. Journal of Theoretical Biology, 110, 275-297.

Perrins, C. 1968. The purpose of high-intensity alarm calls in small passerines. Ibis, 110, 200-201.

Randall, J. A. \& Matocq, M. D. 1997. Why do kangaroo rats (Dipodomys spectabilis) footdrum at snakes? Behavioral Ecology, 8, 404-413.

Randall, J. A. \& Stevens, C. M. 1987. Footdrumming and other antipredator responses in the bannertail kangaroo rat. Behavioral Ecology and Sociobiology, 20, 187-194.

Smythe, N. 1970. On the existence of 'pursuit invitation' signals in mammals. American Naturalist, 104, 491-494.

Vega-Redondo, F. \& Hasson, O. 1993. A game-theoretic model of predator-prey signaling. Journal of Theoretical Biology, 162, 309319.

Woodland, D. J., Jaafar, Z. \& Knight, M. L. 1980. The 'pursuit deterrent' function of alarm signals. American Naturalist, 115, 748.

Yachi, S. 1995. How can honest signalling evolve? The role of handicap principle. Proceedings of the Royal Society of London, Series B, 262, 283-288.

Zahavi, A. 1977. The cost of honesty (Further remarks on the handicap principle). Journal of Theoretical Biology, 67, 603-605.

Zahavi, A. 1987. The theory of signal selection and some of its implications. In: International Symposium on Biology and Evolution (Ed. by V. P. Defino), pp. 305-327. Bari: Adriatica Editrice.

Zahavi, A. 1990. Arabian babblers: the quest for social status in a cooperative breeder. In: Cooperative Breeding in Birds: Long-term Studies of Ecology and Behaviour (Ed. by P. B. Stacey \& W. D. Koenig), pp. 105-130. Cambridge: Cambridge University Press.

Zahavi, A. \& Zahavi, A. 1997. The Handicap Principle. Oxford: Oxford University Press.

Zuberbühler, K., Jenny, D. \& Bshary, R. 1999. The predator deterrence function of primate alarm calls. Ethology, 105, 477-490.

\section{Appendix 1}

\section{Variables}

$t \quad$ Probability that prey is caught if chased (a random variable).

$x$ Observed predation risk (another random variable).

$X_{+}, X_{-}$Set of prey that signal, and do not signal, respectively.

$p(x) \quad$ Conditional probability that the predator is present given observed predation risk $x$.

$\psi(x) \quad$ Probability that the prey will be chased and caught, if the predator is present and chooses to pursue. 
$t^{\star}(x) \quad$ The expected value of $t$ given, when the predator is present.

$t_{+}^{\diamond} \quad$ The expected value of $t$ for prey in $X_{+}$, when the signalling threshold is $x$ and the predator is present.

$t_{-}^{\diamond} \quad$ The expected value of $t$ for prey in $X_{-}$, when the signalling threshold is $x$ and the predator is present.

$\hat{x} \quad$ Signalling threshold: prey signal only if they observe $x>\hat{x}$.

\section{Game parameters}

$f(t, x)$ Joint probability density of $t$ and $x$ when predator is present.

$g(x) \quad$ Probability density of $x$ when predator is absent.

$\alpha \quad$ Probability that the predator is present.

c Cost to the prey of signalling.

d Cost to the predator of pursuit.

\section{Appendix 2: Deriving (8)}

Because $\psi(x)$ is monotone increasing, it follows from inequality (7) that at signalling equilibrium,

$$
\begin{aligned}
& \frac{\int_{0}^{\hat{x}} f_{X}(x)(\psi(\hat{x}) / p(x)) d x}{\int_{0}^{\hat{x}} f_{X}(x) d x}>\frac{\int_{0}^{\hat{x}} f_{X}(x)(\psi(x) / p(x)) d x}{\int_{\hat{x}}^{\hat{x}} f_{X}(x) d x} \\
& \\
&>\frac{\int_{\hat{x}}^{1} f_{X}(x)(\psi(x) / p(x)) d x}{\int_{\hat{x}}^{1} f_{X}(x) d x} \\
& \int_{\hat{x}}^{1} f_{X}(x) d x
\end{aligned} .
$$

Therefore

$$
\frac{\int_{0}^{\hat{x}} f_{X}(x)(\psi(\hat{x}) / p(x)) d x}{\int_{0}^{\hat{x}} f_{X}(x) d x}>\frac{\int_{\hat{x}}^{1} f_{X}(x)(\psi(\hat{x}) / p(x)) d x}{\int_{\hat{x}}^{1} f_{X}(x) d x}
$$

Dividing by $\psi(\hat{x})$, expression (8) follows immediately.

\section{Appendix 3: Sufficient Conditions}

Necessary conditions for equilibrium do not establish existence; in this appendix, we establish the existence of signalling equilibrium by providing a set of sufficient conditions. The conditions here are overly restrictive in the sense that not all signalling equilibria will meet these conditions.

(1) The function $t^{*}(x)$ is continuous and monotone decreasing.

(2) The predator would not pursue the most aware prey but would pursue in the absence of any information about awareness: $t_{+}^{\diamond}(1)<d<t_{+}^{\diamond}(0)$.

From (1), (5), and the first condition above it follows that both $t_{+}^{\diamond}$ and $t_{-}^{\diamond}$ are monotone-decreasing continuous functions. By the second condition above $t_{+}^{\diamond}$ crosses $d$ at exactly one point $\underline{x}$, which will be on the interior of $[0,1]$. Since $\psi$ is continuous and monotone increasing, there will be a nonempty region $(\psi(\underline{x}), \psi(1))$ of signal costs $c$ which allow a signalling equilibrium. Note that this line of argument also establishes the robustness of at least those signalling equilibria which can be constructed in this fashion. Because of the continuous nature of the functions involved and the compact region in which signalling is possible, small changes in the parameters of the system will not cause signalling equilibria in the interior of the signalling region to collapse. 